\title{
APPROACHES TO ENHANCE CNS DELIVERY OF DRUGS ACROSS THE BRAIN BARRIERS
}

\author{
Boppudi.Vinoothna \\ Dept. of Bio Medical Engineering \\ Saveetha School of Engineering, \\ Saveetha University
}

\begin{abstract}
Despite the fact that numerous specialists have helpful possibilities for Central Nervous System (CNS) ailments, few these operators have been clinically utilized in view of cerebrum hindrances. As the defensive hindrance of Central Nervous System (CNS), the Blood Brain Barrier(BBB) and Blood Cerebro Spinal Fluid Barrier(BCSFB) maintains the brain microenvironment, neuronal action, and appropriate working of the Central Nervous System(CNS). The Blood Brain Barrier acts viably to shield cerebrum from flowing pathogens. Antibodies are too huge to even consider crossing the blood mind barrier, and just certain anti-infection agents can pass. In a few cases, a medication needs to regulated straightforwardly into the cerebrospinal fluid(CSF) where it can enter the brain by intersection. The bloodcerebrospinal fluid barrier. Similar to blood brain barrier the blood CSF barrier capacities to forestall the entry of most blood borne substances into cerebrum which specifically allowing the section of explicit substances into brain and encouraging the evacuation of brain metabolites and metabolic items into the blood. Different techniques for effective Central Nervous System (CNS) delivery have been contemplated. This article surveys the present ways to deal with open (or) encourage entrance over these obstructions for improved medication delivery to Central Nervous System (CNS). These methodologies are outlined into three general classifications: Non-invasive, invasive and miscellaneous techniques. The advances made utilizing these methodologies are assessed, and the related systems and issues are talked about in this review article.
\end{abstract}

Keywords: CNS, Drugs, Barriers, brain

\section{INTRODUCTION}

The Blood-Brain barrier (BBB) is a shockingly specific semi permeable fringe as separates the coursing blood from the cerebrum or extracellular liquid in the Central Nervous System(CNS). The Blood Brain boundary is fashioned by means of endothelial cells over the capillary wall, astrocyte end-feet ensheathing the slender, and pericytes fixed solidly in an encompassing mass into the capillary basement membrane. These systems thus allows the movements of some molecules by the passive diffusion. BBB controls the compounds

\author{
Ieshitha Pan \\ Dept. of Bio Medical Engineering \\ Saveetha School of Engineering \\ Saveetha University
}

entering into brain and regulate cerebrum homeostasis. It limits the entrance in similarity with brain cells of blood-borne mixes then encourages supplements fundamental for metabolism to arrive at synapses. It is assessed that over $98 \%$ over little atomic estimation drugs (for the most part peptides yet proteins) thrived for CNS pathologies don't pass the BBB. The ternary functions of BBB include stabilizer-balance out CNS neurons and Protection-from toxins, microbes and Holder-hold synapse inside CNS. The blood-cerebrospinal liquid hindrance (BCSFB) is a liquid mind obstruction that is made out of a couple of films that different blood from CSF at the slim level and CSF from cerebrum tissue. The blood-CSF limit at the choroid plexus is a layer made up of epithelial cells and tight intersections that connect them. There is a CSF-brain boundary at the degree of the piamater, yet just in the embryo. Fundamental medication treatment of neurological sicknesses, for example, mind tumors, natural metabolic blunders (e.g., lysosomal capacity illnesses), and irresistible and neurodegenerative ailments, is a scary errand because of the one of a kind and exceptional defensive obstructions of the focal sensory system (CNS).[1,2] Such intrinsic hindrances, generally the blood-cerebrum boundary (BBB) and the bloodcerebrospinal liquid (CSF) boundary (BCSFB), not just assume a basic job in ensuring the CNS against dangerous and infectious agents while keeping up the ionic and volumetric conditions, yet in addition forestalls the compelling foundational sedate conveyance to the CNS. The BBB comprises of the tight intersections (TJs) of slim endothelial cells on one side and the foot procedures of astrocytes on the opposite side, while the BCSFB is framed by the TJs of choroid plexus cells encompassing the micro vascular endothelium with intracellular hole and fenestration. In spite of the fact that the BBB and BCSFB apply diverse chief capacities as far as the basic difference. $[3,4]$ the two of them take an interest in controlling the exchange of particles between the blood and cerebrum parenchyma or CSF.

Under the physiological condition, coursing atoms can just access to brain or CSF by utilizing a transcellular course through the slim endothelial cells or choroid plexus cells through both inactive and dynamic transport. Free dissemination is the chief latent pathway. For the most part just lipid dissolvable (lipophilic) atoms with a sub-atomic mass underneath 400-600 Da can experience the cells with TJs. Different atoms can likewise jump through certain 
endogenous transport systems of the cells generally through carrier mediated transport,[5] receptor-interceded transport, or absorptive-interceded transport.[6] With these transport systems, basic supplements including amino acids, glucose, carboxylic acids, and nucleosides can diffuse into the brain. The receptor-interceded transport system encourages transport of macromolecules conjugated with surface receptor ligands, for example, transferrin, lactoferrin, and insulin. Absorptiveintervened transport is a sort of endocytosis incited by the substance with cat ionized ligands or peptides, for example, egg whites.

Active transport is the development of a wide range of particles over a cell layer against its focus inclination with cell vitality utilization (e.g., adenosine triphosphate). Active transport can amass high groupings of atoms in the cell, in which the transporters, for example, P-glycoprotein, multidrug-safe protein, multidrug opposition related protein, framework L-transporters, natural anion transporter, natural cation transporter, monocarboxylate transport framework, concentrative nucleoside transporter, equilibrative nucleoside transporter, and peptide transport framework 1 may participate.[7-9]

Thinking about particular cell structures of defensive obstructions, CNS medicate disclosure needs to blend tranquilize configuration or alteration with the improvement of proficient conveyance frameworks. Medication gain admittance to straightforwardly section into the cerebrum has been widely explored during the most recent decade and has gained stamped ground as of late. In this audit, we coordinate the present discoveries in the field of CNS conveyance of medications. The systems and issues engaged with these techniques are additionally examined $[10,11]$

\section{CURRENT APPROACHES FOR ENHANCED DRUG DELIEVERY TO CNS}

Numerous factors can influence the transport of therapeutics over the Blood Brain Barrier and Blood Cerebro Spinal Fluid Barrier. The factors that influence the therapeutics over the $\mathrm{BBB}$ and the BCSFB incorporate

1) Physiochemical properties of the medications

- Lipophilicity

- Molecular weight

- Molecular charge

- Polymorph

- Chemical structure

- Chemical adaptation

2) Biopharmaceutical and pharmacokinetic factors

- Clearance rate

- Membrane transport

- Metabolism instrument

- Systemic assimilation

3) Dosage structure factors

- Preparation, formulation and extra specialists
- Concentration slope of medication or polymer

- Dissolution rate

- Particle size

- Flexibility

- permeability

By and large, most therapeutics of CNS maladies in customary organization enter the CNS by utilizing the foundational blood course. To arrive at colossal medication fixations at the CNS infirmity destinations, it is basic to raise the fundamental medication levels by means of considerably increasingly alluring use or delayed organization. This every now and again builds the hazard of systemic harmfulness. In this way, scientists search for more approaches to move helpful operators into the CNS without the requirement for expanding the fundamental degrees of these specialists. Up to this point, three general classes - noninvasive, intrusive, and different systems - have been created to upgrade sedate conveyance to the CNS.

\section{A. NON-INVASIVE APPROACHES}

Non-invasive approaches include chemical and biological approaches

\section{* CHEMICAL APPROACHES:}

Chemical approaches relies upon synthetic structure change of medications for improving their unacceptable physiochemical properties and along these lines they change their motivation[12].

\section{LIPOPHILIC ANALOGS}

Since lipid solvency is a key factor in inactive dissemination (passive transport) into the $\mathrm{BBB}$, synthetic adjustment of the medication itself into a progressively lipophilic structure (lipidization) has been sought after. In lipidization, lipid bunches are added to the polar parts of the drug molecules to gather into lipophilic analogs, which have preferable cerebrovascular porousness over the first medication. As per past examinations lipophilic analogs with $\log \mathrm{P}$ esteem in the scope of 1.5-2.5 have great CNS penetrability. A ton of corrosive containing drugs have been investigated by such methodologies.

The principle confinements of lipophilic analogs incorporate low selectivity of medication atoms and poor tissue appropriation. Likewise, the lipidization system requires alteration of lipid-like atoms into the hydrophilic piece of the medication structure. Latent dissemination (passive transport) is commonly considered as the principle system over the BBB for lipophilic particles[13]. Nonetheless, the volume of appropriation is commonly improved with the lipidization of atoms, especially when these molecules can tie plasma proteins in blood dissemination. Also, lipophilic analogs can influence the pace of oxidative digestion by cytochrome P-450 compounds and different chemicals. So the benefits of 
lipophilic analogs for cerebrum conveyance may be balanced by the difference in medicate pharmacokinetic parameters.[14] MECHANISM: Receptor/vector mediated transport Liposome should cover with mannose and afterward it arrives at cerebrum tissue where the mannose coat helps transport. Addition of sulphatide which is a sulfate ester of galactocerebroside to liposome expands the accessibility.

\section{TARGETING}

A nonviral supercoiled plasmid DNA is encapsulated in an interior of an $85 \mathrm{~nm}$ liposome

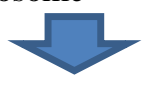

Liposome surface is conjugated with 1000-2000 strands of 2000 Dalton peg to form liposome which is pegylated liposome

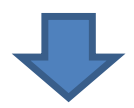

Tips of 1-2\% peg strands are conjugated with a peptidomimetic Mab (HIR/TR) to form pegylated to form immune liposomes

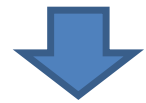

Transfer via RMT

\section{PRODRUGS:}

Prodrugs are characterized as exacerbates that, on organization, must experience compound change by metabolic procedures before turning into a functioning pharmacological agent[12]With synthetic adjustment, the prodrug technique is utilized to make a medication progressively lipophilic. For instance, morphine can't enter the CNS independent from anyone else. After lamentation through acetylating of both hydroxyl gatherings, morphine can undoubtedly cross the BBB and arrive at a viable focus in brain.[13] Be that as it may, care ought to be taken before utilizing the prodrug strategy since some prodrug particles may adjust the first tissue circulation, and the adequacy and poisonous quality of the parent tranquilize. Prodrug is lipid dissolvable when it crosses the $\mathrm{BBB}$ it is used inside the cerebrum and changed over to parent sedate. The medication covalently connected to an idle compound moiety. It improve physiochemical property, for example, solvency and layer porousness. Prodrug is cut by hydrolytic or enzymatic procedures. The drug covalently linked to an inert chemical moiety. It improve physiochemical property such as solubility and membrane permeability. Prodrug is cleaved by hydrolytic or enzymatic processes. Examples include levodopa, gaba, niflumic acid, valproate.

\section{CHEMICAL DRUG DELIVERY SYSTEM:}

The chemical drug delivery system (CDS) term was introduced first by Bodor.[14]Different from the prodrug approach, a CDS typically requires only a single activation step. However, more and more highly developed prodrugs nowadays are activated in multiple steps. Three types of CDS have been most investigated: 1) enzymatic physicochemical CDS, 2) site-specific enzyme-activated CDS, and 3) receptorbased CDS.

\section{MOLECULAR PACKAGING}

To upgrade infiltration of peptides through the BBB, the "atomic bundling" procedure has been created. With atomic bundling, three objectives can be achieved for upgraded BBB entrance: 1) expanded lipophilicity to improve passive transport, 2) anticipation of untimely debasement by expanding enzymatic stability, and 3) exploitation of the lockin component to give focusing on. In sub-atomic bundling, the peptide unit is a piece of a cumbersome particle, commanded by the gatherings that forestall acknowledgment by peptidases and direct BBB penetration.[4]

\section{* BIOLOGICAL APPROACHES}

\section{RECEPTOR VECTOR MEDIATED DELIEVERY OF CHIMERIC PEPTIDES}

The receptor/vector-interceded conveyance of unrealistic peptides procedure depends on utilizing the coupling of a nontransportable peptide pharmaceutical to a transportable peptide or protein, which experiences receptor-intervened or absorptive-intervened transcytosis through the BBB.[22]

Endocytosis can be activated subsequent to binding of the vector to its receptor on the luminal surface of brain capillary endothelial cells. Following movement of the peptide over the cells and exocytosis at the albuminal plasma layer, the peptide is discharged into brain interstitial space. Enzymatic cleavage may happen at the cleavage linkage between the vector and the medication to discharge the pharmacologically dynamic moiety of the fanciful peptide.

The proficiency of receptor-intervened transcytosis over the $\mathrm{BBB}$ can be controlled by tuning the nanoparticle (NP) avidity to be focused on the receptor.[15]In Wiley et al's investigation utilizing gold NPs designed with transferrin,[16]it was discovered that the cerebrum gathering of the NPs was reliant on transferrin level. NPs enriched with a significant level of transferrin particles remain firmly joined to the brain endothelial cells without solid transcytosis, while those with less transferrin can interface with the transferrin receptors on the luminal side of the BBB and withdraw at the cerebrum side to arrive at the brain parenchyma tissues. This examination shows that legitimate enhancement of the energy.

\section{CELL-PENETRATING PEPTIDE (CPP)-MEDIATED DRUG DELIEVERY}

Another technique includes the use of CPPs to enhance the CNS delivery. [25] CPPs comprise a sequence of highly basic 
amino acids which confer a superb charge on the peptide. They interact with the mobile surface by way of a receptor unbiased mechanism. Furthermore, CPPs can transport the molecules that are tagged to them throughout the cell membrane, into the cytoplasm and to the nucleus, [26,27] and this impact is unbiased of the mobile type.

The most regularly studied peptides consist of HIV-1 (human immunodeficiency virus [HIV] kind 1) trans-activating transcriptional activator (TAT) peptide, herpes simplex virus (HSV) type-1 transcription aspect (HSV VP-22) peptide, antennapedia, and penetratin. HIV-TAT peptide was once found in 1988, when researchers determined that TAT protein is able to migrate from the quiescently contaminated cells that produce this protein to the uninfected cells and provoke viral replication. TAT protein incorporates the sequence regarded as protein transduction domain that is responsible for this translocation

TAT peptide is a pretty small polypeptide of 86 amino acids with a cysteine wealthy region. [28] The primary area of TAT peptide consisting of two lysine and six arginine residues is fundamental for efficient mobile uptake. 29] It has been discovered that TAT peptide permeates the cell membrane in a receptor- and transporter-independent mechanism. TAT peptide can permeabilize the cell by forming an inverted micelle, through destabilizing the phospholipids bilayer, by means of interacting with the negatively charged phospholipids of plasma membrane.[30]

Endocytosis and macropinocytosis are the two fundamental mechanisms that have been proposed to explain the cellular uptake of TAT peptide. The main gain of TAT coupling is that, along with environment friendly transport of molecules, biological activity of the coupled molecule is preserved. In addition, the size of the molecule being transported is typically now not a rate limiting factor. TAT conjugation was employed to facilitate the transport of bio macromolecules throughout the BBB.[3]

\section{VIRAL VECTORS}

Viral vectors have become a precious tool for gene shipping at specific site(s) in the brain. Different techniques are employed for the delivery of genes to the brain and enhance their distribution. One of the strategies is to inject the viral vectors without delay into the cerebral lateral ventricles. By this method, virus will be delivered in every part of the CNS.[16] Another approach is to inject at more than one sites to cover a large volume. Agents such as mannitol [17] and heparin[18] have also been examined to promote the distribution of vectors. To date, a variety of virus lessons have been studied as vectors for gene CNS delivery.[19] These consist of HSV,36 lentivirus, [18] retrovirus, recombinant AAV (adenoassociated virus),[20] simian virus [21] and helper-dependent adenovirus.[22] Moreover, hybrid viral vectors have been built to incorporate unlike viral elements with unique features in order to acquire reproducible and stable gene transport to the brain. Various kinds of vectors have been built with the aid of utilizing the aggregate of two or more viral elements or gene sources. Although viral vectors have generally presentment satisfactory efficiency for CNS delivery, they are associated with several problems such as unwanted harm immune response, changes in the properties of delivered virus due to endogenous recombination, and mutagenic behavior leading to oncogenesis. [3,5] It is distinctly recommended for the researchers to weigh the risks in opposition to the benefits before selecting this class of transport system.

\section{COLLOIDAL DRUG CARRIERS}

The vesicular frameworks are firmly requested congregations of one or a few concentric lipid bilayer formed, when certain amphiphilic building squares are gone up against with water. They ought to be covered with surfactants like polyoxyethylene or propylene, PEG.

The aim of the colloidal drug carriers are to control the degradation of drug, prevent harmful side effects, increase the availability of drugs at the disease site and slowly degrade, react to stimuli and be site-specific.

They are highly useful to prolong the existence of drug in systemic circulation, improves the bio availability especially of poorly soluble drugs. Both hydrophilic and lipophilic drugs can be incorporated and also delays the elimination of rapidly metabolizable drugs and this functions as sustained release systems.[23]

\section{NANOPARTICLES}

Nanoparticles size include 1-1000nm that includes both nanocapsules, with a core shell structure and nanospheres.NP transport throughout the BBB may additionally be diminished due to intracellular accumulation at the endothelial cells as giant aggregates. This problem can be addressed the use of the "shuttle mediated" transport strategy. Guarnieri et al these days validated that by using conjugating NPs with a viral fusion peptide (gH625) derived from the glycoprotein $\mathrm{gH}$ of $\mathrm{HSV}$, the aforementioned tendency of forming NP aggregates inside the cells is decreased, and increased BBB transport is subsequently noticed. This is a noteworthy finding as most researchers tend to focus on growing the NP binding on the endothelial cell surface, with much less attention paid to what takes place after the binding. The NPs may also actually get caught on the cell surface or interior the cells, barring actual improvement in the $\mathrm{BBB}$ passage for the desired brain targeting. [24,25]

\section{MECHANISMS OF TRANSPORT}

ADHESION

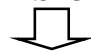

FLUIDIZATION OF BBB ENDOTHELIUM BY SURFACTANTS

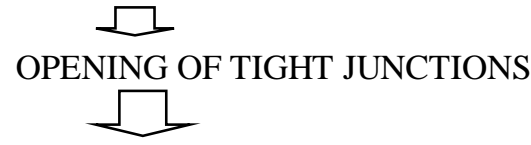




\section{TRANSYTOSIS}

\section{BLOCKAGE OF GLYCOPROTEIN}

\section{B. INVASIVE TECHNIQUES}

By and large, just a couple of peptides and supplements can cross the BBB to accomplish successful fixation inside the brain tissue following intravenous or oral administration. [26] To convey adequate measures of profoundly intense medications (eg, anticancer medications and neurotrophic factors) to the CNS by fundamental courses, it is inescapable these medications will circulate in different tissues to cause genuine dangerous reactions. Now and then, it is important to disturb the BBB or oversee these medications straightforwardly into the cerebrum tissue.

\section{INTRACEREBRAL IMPLANTS}

It comprises of delivery of medications into cerebrum parenchymal surface and the medications can be managed by direct infusion by means of intrathecal catheter and control discharge frameworks and microencapsulated chemicals. The essential mechanism is diffusion.It is helpful in the treatment of various CNS sicknesses for example mind tumor, parkinson's ailment etc, Example: intrathecal infusion of baclofen for spasticity.

Intracerebral implantation of therapeutic agents containing biodegradable polymeric framework or reservoir is an exceptionally horrible medication conveyance technique, which has been used in various clinical preliminaries. In 1996, the FDA affirmed a BCNU (bischloroethylnitrosourea) (carmustine)- contained polyanhydride polymer wafer for repetitive high-grade gliomas.[27]With a mix of dissemination and hydrolytic polymer debasement, this lattice keeps up a continued medication discharge for around 2 months.

\section{INTRA CEREBRO VENTRICULAR INFUSION}

The most immediate method for going around the BBB is to convey tranquilizes legitimately to the intra ventricular, intra cavitary, or interstitial framework. With irregular bolus infusions, these neighborhood conveyance techniques can hypothetically yield high medication fixations in the CNS with negligible foundational presentation and harmfulness. By managing anticancer medications locally to an intracranial objective, continued intracranial medication focuses can be achieved. These conveyance procedures are viewed as the most engaging methods for the treatment of essential brain tumors, since they give the most immediate course to defeat the boundaries to tumor sedate conveyance. A few medications, for example, nitrosourea and methotrexate, have been utilized in different clinical preliminaries with promising outcomes. Be that as it may, this procedure has certain hindrances, including CNS disease, catheter impediment, and lacking medication distribution.[28]
Pharmacological impact is checked whether the objective receptors of the medication are situated close the ependymal surface of the brain. Drug is injected utilizing an ommaya reservoir, a plastic reservoir embedded subcutaneously in the scalp and associated with ventricles. Limitations of this system are that the dispersion of medication in the brain parenchyma is low. Unless the objective is near the ventricles it's anything but a productive technique for sedate deli every.

\section{BBB DISRUPTION STRATEGIES}

\section{OSMOTICBBBDDISRUPTION}

Transient osmotic disturbance of the BBB, BCSFB, and blood-tumor deterrents can be acknowledged through intrablood vessel mixture of a hyper osmotic agent.[29] Hyper osmolar mannitol is the regularly utilized operator for achieving BBBD, which can enhance the BBB penetrability by methods for inciting the shrinkage of cerebrovascular endothelial cells, likewise creating an interruption of between endothelial TJs for different hours. Besides mannitol, hyperosmolar alternatives of arabinose, lactamide, saline, urea, and radiographic qualification retailers can be utilized to momentarily rupture the BBB. Since its underlying portrayal by Rapport in 1972, the strategy has been utilized in preclinical and even clinical studies. A harming dangerous/restorative proportion is oftentimes found with hyper osmotic BBBD. So this strategy needs to be circumspectly used to stay away from unfortunate neurotoxicity.

\section{CONVECTION ENHANCED DELIEVERY}

CED is a transportation technique to manage restorative dealers immediately into focused ability parenchyma or tissue., CED includes one or additional catheters stereo tactically situated through cranial burr gaps into the mind. Remedial dealers are accordingly managed by methods for micro infusion pump.[30]

Contrasted and typical conveyance techniques, CED has demonstrated its interesting attributes for CNS delivery. Without atomic weight impediment, CED can supply two eminent medication fixation in the region of structure into which the catheter is put. Besides, CED inside the portrayed implantation estimations doesn't deliver cerebral edema or quantifiable increments in intracranial strain

\section{BIOCHEMICAL BBBD STRATEGY}

Some vasoactive mixes, including leukotrienes, bradykinin, and histamine, appear to specifically expand the porousness in unusual cerebrum capillaries. [31] Normal brain vessels oppose the impacts of these mixes through an "enzymatic barrier" that may inactivate vasoactive agents conversely, this enzymatic boundary is lost in certain ailing states, enabling vasoactive mixes to specifically build penetrability in strange vessels. Clinically, intra carotid imbuement of leukotrienes, bradykinin, and other vasoactive agents can expand tranquilize 
conveyance to unhealthy tissue. In contrast to osmotic disturbance strategies, biochemical BBBD is a less intrusive and potentially an increasingly dependable procedure for BBBD in light of the fact that it chiefly influences the sick vasculature.

\section{CONCLUSION}

With the fast advancement of present day society and the proceeded with decay of the earth, brain sicknesses, for example, neurodegenerative infection (eg, Alzheimer's illness and Parkinson's ailment), stroke, neuro inflammation, and neuro-oncology have become distressful and in any event, pulverizing issues for individuals. Drug delivery throughout the physiological obstacles of the intelligence is the bottleneck because the therapy about CNS disorders then brain tumors. The delivery about most capsules in conformity with the CNS is constrained through the bodily shape at the BBB yet BCSFB. In this article, some promising strategies for environment friendly CNS transport were reviewed. Although most of these approaches still stay in the experimental stage, persevering with effort in acquiring improved grasp of the mechanisms involved and developing new CNS delivery procedures will facilitate faster translation and sizable adoption of these methods in clinical applications. While modern studies have made exceptional progress, it is well worth noting that medical translation of BBB opening approach is nonetheless associated with a number of concerns. The first problem is the reproducibility and feasibility of these BBB opening strategies. Although some encouraging results had been bought in animal experiments, the complexity of the marketers or settings used in various strategies would limit their future applications. For example, floor modified nano scale particles have their benefits in BBB opening strategies, however most of these nano scale particles are self-made agents. Strictly speaking, the data from experiments the uses of the identical method are not comparable because the components in these sellers are different. Even for these dealers composed of the same components, the number characteristics resulting from their one of a kind preparations or floor modifications still affect the accuracy of the results. Finally, while exciting results hold been learnt yet manipulative techniques have been mounted between animals, that should stay pointed out so much these BBB beginning strategies have only been established in conformity with lie feasible among awful experiments. The physiological structure over the BBB is no longer the same of primates. Thus, the consequences present out of rodents may additionally now not keep applicable in imitation of humans, or investigations among primates including stringent experimental designs then medical trials are required before these techniques can remain utilized because of the cure on ethnical brain diseases. After top addressing these issues, nano carriers blended with medical imaging or BBBD techniques might also pave the road in imitation of successfully do along a widespread range To conclude, centered CNS therapeutics is an early and late expanding and challenging however promising field. Design or development on superb focused CNS therapeutics requires thoroughgoing appreciation on the physiochemical residences of the drugs, biopharmaceutical or pharmacokinetic job over the carriers, as much nicely as much the organic parameters of the CNS. Further insights of the molecular mechanisms involved in $\mathrm{BBB}$ and $\mathrm{BCSFB}$ regulation will provide additional molecular cues for more selective and efficient targeting of the brain barriers in CNS diseases.

\section{ACKNOWLEDGEMENT}

I have prepared this paper with the help of many books, websites etc. I pay my sincere gratitude to all the authors, professors, experts for their efforts and contributions. I thank anonymous referees for their useful suggestions.

\section{REFERENCE}

1. Begley DJ. (2004) Delivery of therapeutic agents to the central nervous system: the problems and the possibilities. (pg.04:29-45.)

2. Chen Y, Halwadi G, Benson HA. (2004) Drug delivery across the blood-brain barrier. Current Drug Delivery(pp.1:361-376.)

3. Madrid Y, Langer LF, Brem H, et al (1991). New directions in the delivery of drugs and other substances to the central nervous system (pg22:29932).

4. Dwibhashyam VS, Nagappa AN.(2008) Strategies for enhanced drug delivery to the central nervous system.(pp.70:145-153).

5. Vykhodtseva N, McDannold N, Hynynen K.(2008) Progress and problems in the application of focused ultrasound for blood-brain barrier disruption. Ultrasonic's ;( pp.48:279-296).

6. Sai Y, Kajita M, Tamai I, et al (1998). Adsorptivemediated endocytosis of a basic peptide in enterocyte-like Caco-2 cells. Am J Physiol; ( pp.275: G514-G520).

7. Tamai I, Tsuji A.(2000) Transporter-mediated permeation of drugs across the blood-brain barrier. $J$ Pharm Sci ; (pg.89:1371-1388).

8. Tsuji A. (2005) Small molecular drug transfer across the blood-brain barrier via carrier-mediated transport systems. NeuroRx;( pp.2:54-62).

9. Schinkel AH. (2001) The roles of P-glycoprotein and MRP1 in the blood-brain and blood-cerebrospinal fluid barriers.AdvExp Med Biol ; ( pp.500:365-372).

10. Thiebaut F, Tsuruo $T$, Hamada H, et al.(2005) Immuno histochemical localization in normal tissues of different epitopes in the multidrug transport;(pg.89:1371-1388).

11. Banks WA, Kastin AJ, Ehrensing CA.(1993) Endogenous peptide Tyr-Pro-Trp-Gly-NH2 (Tyr-WMIF-1) is transported from the brain to the blood by 


\section{International Journal of Engineering Applied Sciences and Technology, 2020 \\ Vol. 4, Issue 12, ISSN No. 2455-2143, Pages 347-353 \\ Published Online April 2020 in IJEAST (http://www.ijeast.com)}

peptide transport system-1. J Neurosci Res ; ( pp.35: 690-695).

12. Albert A.(1958) Chemical aspects of selective toxicity. Nature ; (pp.182: 421-422).

13. Pardridge WM. (1998) Recent advances in bloodbrain barrier transport. Annu Rev Pharmacol Toxicol ;( pp.28:25-39).

14. Bodor N, Buchwald P.(1999) Recent advances in the brain targeting of neuro pharmaceuticals by chemical delivery systems. Adv Drug Deliver Rev ; pp.36:229-254).

15. Brewster ME, Anderson WR, Webb AI, et al. (1997) Evaluation of a brain-targeting zidovudine chemical delivery system in dogs. Antimicrobial Agents Chemotherapy ;( pg.41:122-128).

16. Brewster ME, Raghavan K, Pop E, et al.(1994) Enhanced delivery of ganciclovir to the brain through the use of redo targeting. Antimicrobial Agents Chemotherapy;( pp.38:817-823).

17. Wu WM, Pop E, Shek E, et al.(1990) Brain and CSF specific chemical delivery systems for beta-lactam antibiotics;(pp.7:33-43).

18. Sikic BI, Fisher GA, Lum BL, et al.(1997) Modulation and prevention of multidrug resistance by inhibitors of P-glycoprotein. Cancer ChemotherPharmacol; (Suppl: S13-S19).

19. Bodor N, Buchwald P.(2002) Barriers to remember: brain-targeting chemical delivery systems and Alzheimer's disease. Drug Discovery Today; (pp.7:766-774).

20. Petri B, Bootz A, Khalansky A, et al.(2007) Chemotherapy of brain tumor using doxorubicin bound to surfactant-coated poly(butyl cyanoacrylate) nanoparticles: revisiting the role of surfactants;(pp.117:51-58).

21. Bodor N, Prokai L, Wu WM, et al.(1992) A strategy for delivering peptides into the central nervous system by sequential metabolism. (pp.257:16981700).

22. Kravcik S, Gallicano K, Roth V, et al.(1999) Cerebrospinal fluid HIV RNA and drug levels with combination of sequin virus. J Acquired Immune Deficiency Syndrome; (pp.21:371-375).

23. Lindgren $M$, Hall brink $M$, Prochiantz A, et al.(2000) Cell-penetrating peptides. Trends Pharmacology Sci; (pp.21:99-103).

24. Misra A, Ganesh S, Shahiwala A, et al.(2003) Drug delivery to the central nervous system: a review. Sci; (pp.6:252-273).

25. Kumar A, Singh TD, Singh SK, et al.(2009) Methods, potentials, and limitations of gene delivery to regenerate central nervous system cells. Biologics; (pp.3:245-256).
26. Wolfe D, Goins WF, Yamada M, et al.(1999) Engineering herpes simplex virus vectors for CNS applications. Exp Neural; (pp.159: 34-46).

27. Graters M, Edgemont K, Hernandez-Acosta P, et al. (2006) Lentiviral vectors mediate efficient and stable gene transfer in adult neural stem cells in vivo. Hum Gene Therapy; (pp.17:635-650).

28. Cepko CL, Ryder E, Austin C, et al. (1999) Lineage analysis using retroviral vectors. Methods. (pp.14:393-406).

29. Mori S, Wang L, Takeuchi T, et al.(2004) Two novel adeno-associated viruses from cynomolgus monkey: pseudo typing characterization of capsid protein. Virology. (pp.330:375-383).

30. Louboutin JP, Reyes BA, Agrawal L, et al.(2001) Strategies for CNS-directed gene delivery: in vivo gene transfer to the brain using SV40-derived vectors. Gene Therapy. (pp.14:939-949).

31. Strayer DS, Agrawal L, Cordelier P, et al. (2006) Long-term gene expression in dividing and non dividing cells using SV40-derived vectors. Mol Biotechnology ;( pp.34:257-270) 